\title{
Interplay between Lattice Distortions, Vibrations and Phase Stability in NbMoTaW High Entropy Alloys
}

\author{
Fritz Körmann * and Marcel H.F. Sluiter
}

Department of Materials Science and Engineering, Delft University of Technology, Mekelweg 2, 2628 CD Delft, The Netherlands; M.H.F.Sluiter@tudelft.nl

* Correspondence: f.h.w.kormann@tudelft.nl; Tel.: +31-15-278-1972

Academic Editor: An-Chou Yeh

Received: 26 July 2016; Accepted: 18 August 2016; Published: 20 August 2016

\begin{abstract}
Refractory high entropy alloys (HEA), such as BCC NbMoTaW, represent a promising materials class for next-generation high-temperature applications, due to their extraordinary mechanical properties. A characteristic feature of HEAs is the formation of single-phase solid solutions. For BCC NbMoTaW, recent computational studies revealed, however, a $\mathrm{B} 2(\mathrm{Mo}, \mathrm{W} ; \mathrm{Nb}, \mathrm{Ta})$-ordering at ambient temperature. This ordering could impact many materials properties, such as thermodynamic, mechanical, or diffusion properties, and hence be of relevance for practical applications. In this work, we theoretically address how the B2-ordering impacts thermodynamic properties of BCC NbMoTaW and how the predicted ordering temperature itself is affected by vibrations, electronic excitations, lattice distortions, and relaxation energies.
\end{abstract}

Keywords: high entropy alloy; ordering; lattice distortions; vibrations; density functional theory

\section{Introduction}

Refractory high entropy alloys (HEAs) such as BCC NbMoTaW are of great interest due to their extraordinary mechanical properties [1-6]. Characteristic of HEAs is the lack of ordering commonly attributed to the large configurational entropy [7-9]. For BCC NbMoTaW, no signature of ordering has been experimentally found in the annealed state or at room temperature [3]. However, thermodynamic equilibrium at experimental conditions is limited due to slow diffusion inherent in refractory alloys. Recent theoretical works have reported a B2 ordering between mixed $(\mathrm{Mo}, \mathrm{W})$ and $(\mathrm{Nb}, \mathrm{Ta})$ sites at ambient temperatures [10-14]. A recent first-principles study combining chemical interactions from the generalized perturbation method with Monte Carlo simulations revealed an ordering temperature of about $750 \mathrm{~K}$ [15]. However, lattice distortions as well as vibrations (phonons) have not been taken into account within this approach, and their impact on the order-disorder temperature remains unknown. Furthermore, the B2 ordering could also impact lattice excitations, and hence thermodynamic properties such as the lattice expansion and the heat capacity.

In this work, we close the previous simulation gap. We theoretically address the question of how local relaxations as well as vibrational and electronic entropies impact the theoretical B2 ordering temperature. We also report how the B2 ordering affects the heat capacity, thermal expansion, and bulk modulus of the alloy.

\section{Materials and Methods}

Density functional theory calculations have been carried out employing the VASP code [16,17] and the projector-augmented wave (PAW) method [18] within the generalized gradient approximation [19]. The semi core $p$ states were treated as valence within the PAW potentials. The planewave cutoff was set to $400 \mathrm{eV}$. Chemical disorder was simulated utilizing the concept of special quasi-random structures (SQS) [20] for 128 atom supercells $(4 \times 4 \times 4$ unit cells). For B2, Mo 
and $\mathrm{W}(\mathrm{Nb}$ and $\mathrm{Ta}$ ) are randomly distributed on the first (second) sublattice. The employed SQS have been constructed by minimizing the correlation functions of the first two nearest-neighbor shells. The employed 128 atom cells provide a fair compromise between mimicking chemical disorder within a limited supercell size and computational efficiency. For a recent discussion on the application of SQS for HEAs, we refer to [21]. A $4 \times 4 \times 4 \Gamma$-centered $k$-point mesh and the Methfessel-Paxton method (order 1 ) with a smearing parameter of $0.1 \mathrm{eV}$ were chosen for the total energy calculations. To study the impact of lattice expansion, all calculations were performed at nine different lattice constants around the theoretical equilibrium. The cubic cell shape was fixed, while the internal atom positions have been fully relaxed.

Ground state volume, bulk modulus, and its derivative were obtained by parametrizing the computed ground state energies, employing Vinet's equation of state [22]. The electronic free energy is derived by occupying the electronic density of states with the Fermi-Dirac distribution. The Gibbs energy due to vibrations is computed by utilizing the Debye-Grüneisen model [23], as explained in detail in [24].

\section{Results and Discussion}

Based on the previous findings, we constructed two supercells (shown in Figure 1) for the A2 (solid solution) in (a) and (b), as well as for the B2 phase (d) and (e). For both structures, we computed the total energies

$$
E_{\text {tot }}^{\sigma}(V)=E_{\text {static }}^{\sigma}(V)+\Delta E_{\text {relax }}^{\sigma}(V)
$$

where $\sigma$ indicates the $\mathrm{A} 2$ and $\mathrm{B} 2$ phase. As we are interested in the impact of internal atomic relaxations, the total energy above is split into the contributions arising from the static bcc lattice, $E_{\text {static }}^{\sigma}$ and the energy contribution due to internal relaxations, denoted as $\Delta E_{\text {relax }}^{\sigma}$. Indeed, lattice distortions constitute one of the main features in HEAs [7-9], and have been investigated, for example, for the FeCoNiCrMn (Cantor) HEA recently [25]. For binary alloys, the importance of relaxation energies to phase stability computations have been recognized some time ago (e.g., [26-28]). For the here-considered refractory HEA, a similar analysis is, however, lacking so far.

The results for $E_{\text {tot }}^{\sigma}(V)$ for the A2 and B2 phases are shown in Figure 1c,f, respectively. We first notice that the B2 phase is lower in energy at zero $\mathrm{K}$, compared to the A2 solid solution. This is consistent with the B2 ordering at ambient temperatures found in previous works [10-15]. Considering the impact of atomic relaxations, we find that the A2 total energy is lowered by $\approx 12 \mathrm{meV}$ per atom (difference between red line/circles and black line/squares in Figure 1c) whereas there is only very little impact of atomic relaxations on the B2 total energy $(<1 \mathrm{meV})$ shown in Figure 1f. The relaxation energies are also summarized in Table 1. The rather small impact of atomic relaxations and relaxation energies can be intuited by examining the B2 ordered phase in Figure 1e. The two sublattices are occupied by larger $(\mathrm{Nb}, \mathrm{Ta})$ and smaller $(\mathrm{Mo}, \mathrm{W})$ atoms, shown in blue and red, respectively. Note that the atomic sizes of the larger $\mathrm{Nb}$ and $\mathrm{Ta}$ atoms (dark and light blue) are exaggerated to facilitate the visualization. From the atomic arrangement of larger and smaller atoms occupying distinct sublattices, only very little relaxations are indeed expected for the B2 phase. In contrast to this, the A2 phase shown in Figure 1a,b features a random distribution of larger $(\mathrm{Nb}, \mathrm{Ta})$ and smaller $(\mathrm{Mo}, \mathrm{W})$ atoms, causing a significantly larger amount of local distortions, and hence relaxation energies. From the different $\Delta E_{\text {relax }}^{\sigma}$ contributions for A2 and B2, we can conclude that local atomic relaxations will have an impact on the ordering temperature, which we will discuss below. 


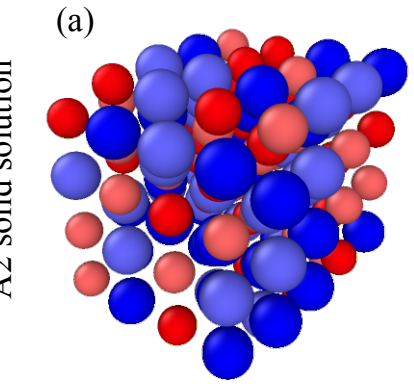

(b)

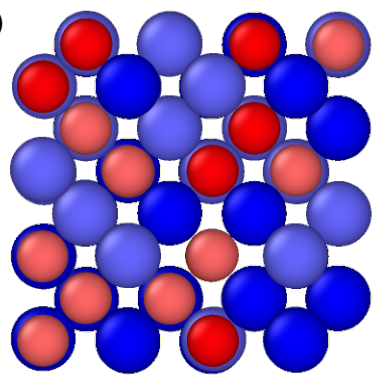

(d)

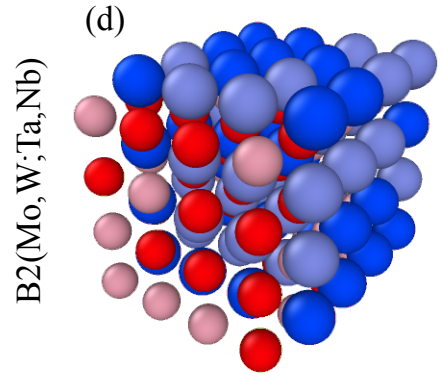

(e)

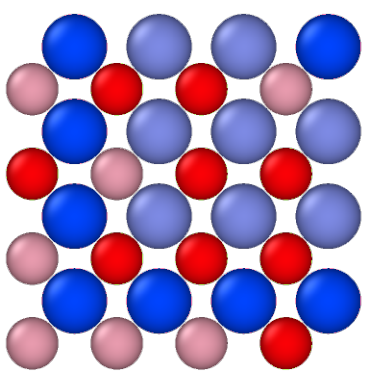

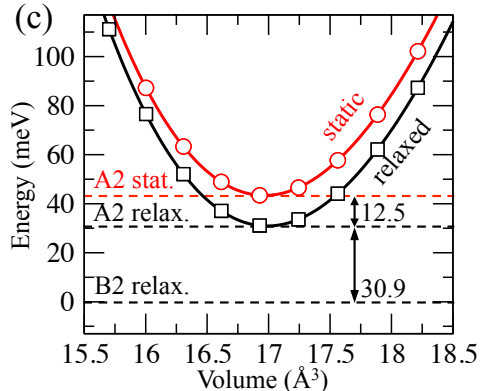

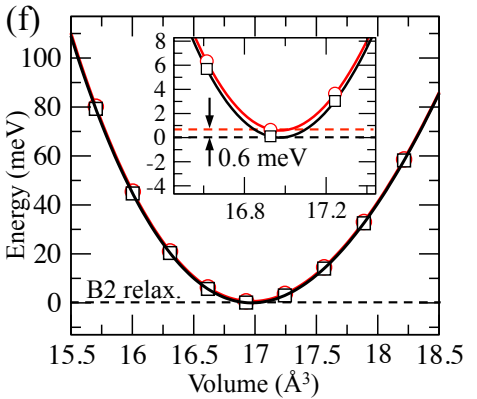

Figure 1. Side and top view on the employed supercells for the high entropy alloy (HEA) in the A2 (a) and (b), as well as for the B2 phase, (d) and (e). To facilitate the visualization the atomic sizes of the larger atoms, $\mathrm{Nb}$ and $\mathrm{Ta}$ (in red and light red) are enhanced compared to the smaller Mo and $\mathrm{W}$ atoms (in blue and light blue). Figures have been created employing the OVITO program package [29]. The computed energy-volume per atom curves for both phases with and without relaxation energy (black and red solid lines) are shown in (c) and (f).

Table 1. Ground state properties, Debye temperatures, and relaxation energies of the NbMoTaW A2 and $\mathrm{B} 2(\mathrm{NbTa} ; \mathrm{MoW})$ phase.

\begin{tabular}{llccccc}
\hline Phase & Method & $\boldsymbol{V}_{\mathbf{0}}\left(\mathbf{A}^{\mathbf{3}}\right)$ & $\boldsymbol{B}_{\mathbf{0}}(\mathrm{GPa})$ & $\boldsymbol{B}^{\prime}$ & $\boldsymbol{\Delta} \boldsymbol{E}_{\text {relax }}(\mathbf{m e V})$ & $\boldsymbol{\Theta}_{\boldsymbol{D}}(\mathbf{K})$ \\
\hline \multirow{2}{*}{ B2(MoW;NbTa) } & PAW + SQS & 16.98 & 233 & 4 & - & 296 \\
& PAW + SQS + relax & 16.97 & 233 & 4 & 0.6 & 296 \\
\hline \multirow{5}{*}{ A2 solid solution } & PAW + SQS & 16.97 & 232 & 4 & - & 296 \\
& PAW + SQS + relax & 16.99 & 231 & 4 & 12.5 & 295 \\
& PAW + SQS + relax [30] & 17.08 & 228 & $\mathrm{n} / \mathrm{a}$ & $\mathrm{n} / \mathrm{a}$ & $\mathrm{n} / \mathrm{a}$ \\
& Experiment [4] & 16.70 & 220 & - & - & - \\
\hline
\end{tabular}

Before discussing the ordering temperature, we first investigate the impact of B2 ordering on ground state and thermodynamic properties. In Table 1, we summarize the ground state volume, $V_{0}$, bulk modulus, $B_{0}$, and its derivative, $B^{\prime}$, derived from the ground state energies in Figure 1 . Although the relaxation energy for A2 is not negligible, the impact on the ground state properties is rather small. This indicates an almost constant and volume-independent $\Delta E_{\text {relax }}^{\sigma}$. We note that our results are also in good agreement with previous calculations [30]. In order to evaluate the impact of B2 ordering on the thermodynamic properties, we compute the Gibbs energies of both phases, $G^{\sigma}(T)$, from which thermodynamic properties such as the volume expansion or heat capacity can be derived.

The Gibbs energies of the B2 and A2 solid solution at ambient pressure are given in the adiabatic approximation as

$$
G^{\sigma}(T)=H_{\mathrm{tot}}^{\sigma}+G_{\mathrm{el}}^{\sigma}(T)+G_{\mathrm{vib}}^{\sigma}(T)-T S_{\mathrm{conf}}^{\sigma}
$$


where $H_{\mathrm{tot}}^{\sigma}$ is the enthalpy, $G_{\mathrm{el}}^{\sigma}(T)$ is the electronic Gibbs energy, $G_{\mathrm{vib}}^{\sigma}(T)$ is the vibration Gibbs energy, and $S_{\text {conf }}^{\sigma}$ denotes the configuration entropy. The ideal configuration entropy for B2 (with disordered $\mathrm{Nb}$, Ta and $\mathrm{Mo}, \mathrm{W}$ on each sublattice) is $S_{\mathrm{conf}}^{\mathrm{B} 2}=\log (2)$, and for the A2 solid solution $S_{\mathrm{conf}}^{\mathrm{A} 2}=\log (4)$. Note that configuration entropy is, in general, temperature dependent (e.g., $\left.[15,31]\right)$, as discussed below.

The vibration contribution, $G_{\text {vib }}^{\sigma}(T)$, is modeled within the quasi-harmonic Debye-Grüneisen model [23], as described in detail in [24]. A similar approach has recently been employed to study thermodynamic properties of other refractory alloys (HfNbZr, HfNbTiZr, and HfNbTaTiZr) [32]. Note that despite recent progress in the computation of phonons for disordered binary alloys [33,34], these methods have not yet been advanced to the stage where they are applicable to multi-componen alloys.

The key ingredient for our Debye model is the Debye temperature, $\Theta_{D}$, which can be derived from the ground state properties. The values for $\Theta_{D}$ are listed in Table 1. For B2 and A2, the same $\Theta_{D}$ of $296 \mathrm{~K}$ is found. Only a marginal reduction of $\approx 1 \mathrm{~K}$ is found for $\Theta_{D}$ of the $\mathrm{A} 2$ when the effects of local relaxations are included.

From Equation (2) we compute for both phases (B2 and A2) the specific heat capacity, $C_{P}(T)$, the volume expansion, $V(T)$, as well as the bulk modulus, $B(T)$. The results for $\mathrm{B} 2$ and $\mathrm{A} 2$ are shown in Figure 2 as black and red solid lines, respectively. The dashed lines show the results without taking electronic excitations into account. For all considered temperatures, the impact of B2 ordering turns out to be negligible. This is consistent with the very similar ground state properties and $\Theta_{D}$ listed in Table 1 derived for both phases. The impact of B2 ordering on the electronic contribution is also negligible, although $G_{\mathrm{el}}^{\sigma}$ is of course important for accurate thermodynamic predictions-e.g., for the specific heat capacity in Figure 2a.
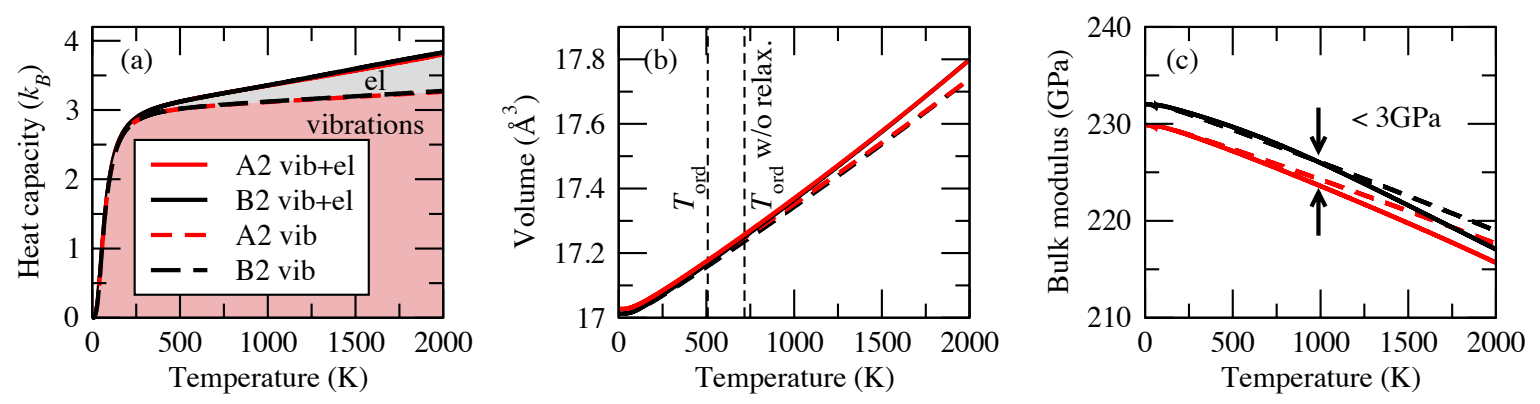

Figure 2. (a) Specific heat capacity, (b) volume expansion, and (c) bulk modulus versus temperature are shown for the $\mathrm{B} 2$ (NbTa;MoW) (black lines) and $\mathrm{A} 2$ (NbMoTaW) solid solution (red lines), with and without (solid/dashed lines) electronic contributions. The predicted B2-ordering temperatures from the Gibbs energy analysis are indicated in (b). The B2 ordering has little impact on thermodynamic properties (see text for details).

We can therefore conclude that the B2 ordering has practically no impact on the lattice parameter or the bulk modulus, and probably not on the elastic properties either. The heat capacities of B2 and A2 do not differ significantly from one another. Note, however, that Figure 2 does not contain the contributions of chemical short-range order, which gives a non-vanishing contribution to the heat capacity around the phase transition.

From the computed Gibbs energies (Equation 2), we can relate the ordering temperature to the Gibbs energy difference between the A2 and B2 phase; i.e.,

$$
\Delta G^{\mathrm{A} 2-\mathrm{B} 2}(T)=G^{\mathrm{A} 2}(T)-G^{\mathrm{B} 2}(T)=\Delta H_{\mathrm{tot}}+\Delta G_{\mathrm{el}}(T)+\Delta G_{\mathrm{vib}}(T)-T \Delta S_{\mathrm{conf}}
$$

From above equation, we estimate the ordering temperature via $\Delta G^{\mathrm{A} 2-\mathrm{B} 2}\left(T_{\text {ord }}\right) \equiv 0$. An advantage of our theoretical approach is that it allows us to "switch on" and "off" different contributions entering Equation (3), and thus to estimate their individual importance on $T_{\text {ord }}$. 
We first assume an ideal bcc lattice without taking local atomic relaxations into account; i.e., $E_{\text {tot }}^{\sigma} \equiv E_{\text {static }}^{\sigma}$ and $\Delta E_{\text {relax }}^{\sigma}=0$. If only configurational entropy in Equation (3) is taken into account, a transition temperature of $717 \mathrm{~K}$ is obtained (see also Table 2), being in good agreement with our previous result of $\approx 750 \mathrm{~K}$ based on the generalized perturbation method derived on the static lattice and Monte Carlo simulations [15]. The slightly lower ordering temperature derived here compared to [15] can be partially attributed to the overestimated ideal configurational entropy $S_{\text {conf }}^{\text {A2 }}$ in the present work. Neither the inclusion of vibrations nor of electronic contributions change the transition temperature. This is consistent with the very similar ground state properties (in particular, bulk modulus and Debye temperature) of the A2 and B2 phase in Table 1 and the negligible impact of B2 ordering on thermodynamic properties in Figure 2.

Table 2. Enthalpy difference at $T=0 \mathrm{~K}$ between $\mathrm{B} 2(\mathrm{NbTa} ; \mathrm{MoW})$ and $\mathrm{A} 2(\mathrm{NbMoTaW})$ solid solution, as well as predicted B2-ordering temperatures if configuration entropy, lattice excitations, and electronic contributions are taken into account.

\begin{tabular}{lcccc}
\hline Method & $\Delta \boldsymbol{H}_{\text {tot }}^{\mathrm{A2}-\mathrm{B} 2}(\mathbf{m e V})$ & $\boldsymbol{T}_{\text {ord }}^{\text {conf }}(\mathbf{K})$ & $\boldsymbol{T}_{\text {ord }}^{\text {conf+vib }}(\mathbf{K})$ & $T_{\text {ord }}^{\text {conf }+ \text { vib+el }}$ \\
\hline PAW + SQS & 42.8 & 717 & 717 & 717 \\
PAW + SQS + relax & 30.9 & 517 & 508 & 508 \\
\hline
\end{tabular}

We now include atomic relaxations, which, as discussed above, lowers the A2 energy by about $12 \mathrm{meV}$ compared to the B2 phase, which has been neglected in [15]. Indeed, if the relaxation energies, $\Delta E_{\text {relax }}^{\sigma}$, are included, the ordering temperature is reduced by almost $30 \%$ down to $517 \mathrm{~K}$. Similar to the unrelaxed scenario, vibrations do not significantly change the ordering temperature $(<10 \mathrm{~K})$, revealing a final predicted ordering temperature of $508 \mathrm{~K}$ if relaxations, vibrations, electronic excitations, and configurational entropy are taken into account. We can therefore conclude that vibrations and electronic excitations are not critical for the phase stability analysis in $\mathrm{NbMoTaW}$ HEAs, while local atomic relaxations and relaxation energies turn out to be significant.

\section{Conclusions}

We studied the A2-B2 ordering in NbMoTaW refractory alloys, in particular the impact of vibrations, electronic excitations, and lattice distortions on the ordering temperature, as well as the impact of B2 ordering on thermodynamic properties. We find that relaxations and B2 ordering have only marginal impact on the ground state volume and bulk modulus. The heat capacity of B2 and A2 differ little, although there is of course a significant contribution due to long and short-range order around the order-disorder temperature. The volume expansion and bulk modulus do not appear to be affected by the B2 ordering.

Based on total energy calculations and ideal configurational entropy, the A2-B2 ordering temperature is found to be in good agreement with previous works. Vibrations are found to play a minor role in determining the ordering temperature. In contrast to the A2 phase, the B2 phase is only slightly affected by atomic relaxations, due to the similar size of $\mathrm{Nb}$ and Ta as well as Mo and $\mathrm{W}$. Regarding the competition of A2 and B2 Gibbs energies, the lattice relaxations turn out to significantly reduce the predicted ordering temperature by almost $30 \%$.

Our results suggest that although thermodynamic properties of the refractory NbMoTaW HEA can be efficiently modeled based on static lattice calculations, accurate ordering temperature computations require the inclusion of local atomic relaxations.

Acknowledgments: Funding by the scholarship KO 5080/1-1 of the Deutsche Forschungsgemeinschaft (DFG) is gratefully acknowledged. We thank Andrei V. Ruban for providing the spcm program for generating the special quasi-random structures. We gratefully acknowledge Blazej Grabowski for stimulating discussions as well as for providing thermodynamic evaluation scripts. 
Author Contributions: Fritz Körmann performed the DFT calculations which were analyzed together with Marcel H.F. Sluiter. Both authors have equally contributed to the overall discussion and preparation of the manuscript as well as read and approved the final manuscript version.

Conflicts of Interest: The authors declare no conflict of interest. The founding sponsor had no role in the design of the study; in the collection, analyses, or interpretation of data; in the writing of the manuscript, and in the decision to publish the results.

\section{References}

1. Yao, H.; Qiao, J.W.; Gao, M.C.; Hawk, J.A.; Ma, S.G.; Zhou, H. MoNbTaV medium-entropy alloy. Entropy 2016, 18, 189.

2. Zou, Y.; Ma, H.; Spolenak, R. Ultrastrong ductile and stable high-entropy alloys at small scales. Nat. Commun. 2015, 6, 7748 .

3. Senkov, O.N.; Wilks, G.B.; Miracle, D.B.; Chuang, C.P.; Liaw, P.K. Refractory high-entropy alloys. Intermetallics 2010, 18, 1758-1765.

4. Senkov, O.N.; Wilks, G.B.; Scott, J.M.; Miracle, D.B. Mechanical properties of $\mathrm{Nb}_{25} \mathrm{Mo}_{25} \mathrm{Ta}_{25} \mathrm{~W}_{25}$ and $\mathrm{V}_{20} \mathrm{Nb}_{20} \mathrm{Mo}_{20} \mathrm{Ta}_{20} \mathrm{~W}_{20}$ refractory high entropy alloys. Intermetallics 2011, 19, 698-706.

5. Maiti, S.; Steurer, W. Structural-disorder and its effect on mechanical properties in single-phase TaNbHfZr high-entropy alloy. Acta Mater. 2016, 106, 87-97.

6. Zou, Y.; Maiti, S.; Steurer, W.; Spolenak, R. Size-dependent plasticity in an $\mathrm{Nb}_{25} \mathrm{Mo}_{25} \mathrm{Ta}_{25} \mathrm{~W}_{25}$ refractory high-entropy alloy. Acta Mater. 2014, 65, 85-97.

7. Murty, B.S.; Yeh, J.W.; Ranganathan, S. High-Entropy Alloys. Butterworth-Heinemann: Oxford, UK, 2014.

8. Zhang, Y.; Zuo, T.T.; Tang, Z.; Gao, M.C.; Dahmen, K.A.; Liaw, P.K.; Lu, Z.P. Microstructures and properties of high-entropy alloys. Prog. Mater. Sci. 2014, 61, 1-93.

9. Gao, M.C.; Yeh, J.W.; Liaw, P.K.; Zhang, Y. High-Entropy Alloys: Fundamentals and Applications, 1st ed.; Springer: Cham, Switzerland, 2016.

10. Huhn, W.P. Thermodynamics from First Principles: Prediction of Phase Diagrams and Materials Properties Using Density Functional Theory. Ph.D. Thesis, Carnegie Mellon University, Pittsburgh, PA, USA, May 2014.

11. Huhn, W.P.; Widom, M. Prediction of A2 to B2 phase transition in the high-entropy alloy Mo-Nb-Ta-W. JOM 2013, 65, 1772-1779.

12. Widom, M.; Huhn, W.P.; Maiti, S.; Steurer, W. Hybrid monte carlo/molecular dynamics simulation of a refractory metal high entropy alloy. Metall. Mater. Trans. A 2014, 45, 196-200.

13. Del Grosso, M.F.; Bozzolo, G.; Mosca, H.O. Determination of the transition to the high entropy regime for alloys of refractory elements. J. Alloy. Compd. 2012, 534, 25-31.

14. Del Grosso, M.; Bozzolo, G.; Mosca, H.O. Modeling of high entropy alloys of refractory elements. Physica B 2012, 407, 3285-3287.

15. Körmann, F.; Ruban, A.V.; Sluiter, M.H. Long-ranged interactions in bcc NbMoTaW high-entropy alloys. Mater. Res. Lett. 2016, 0, 1-6, doi:10.1080/21663831.2016.1198837.

16. Kresse, G.; Furthmüller, J. Efficient iterative schemes for ab initio total-energy calculations using a plane-wave basis set. Phys. Rev. B 1996, 54, 11169.

17. Kresse, G.; Furthmüller, J. Efficiency of ab-initio total energy calculations for metals and semiconductors using a plane-wave basis set. Comput. Mater. Sci. 1996, 6, 15-50.

18. Blöchl, P.E. Projector augmented-wave method. Phys. Rev. B 1994, 50, 17953.

19. Perdew, J.P.; Burke, K.; Ernzerhof, M. Generalized gradient approximation made simple. Phys. Rev. Lett. 1996, 77, 3865.

20. Zunger, A.; Wei, S.H.; Ferreira, L.G.; Bernard, J.E. Special quasirandom structures. Phys. Rev. Lett. 1990, $65,353$.

21. Gao, M.C.; Niu, C.; Jiang, C.; Irving, D.L. Applications of Special Quasi-random Structures to High-Entropy Alloys. In High-Entropy Alloys; Springer: Cham, Switzerland, 2016; pp. 333-368.

22. Vinet, P.; Ferrante, J.; Rose, J.H.; Smith, J.R. Compressibility of solids. J. Geophys. Res. Solid Earth 1987, 92, 9319-9325.

23. Moruzzi, V.L.; Janak, J.F.; Schwarz, K. Calculated thermal properties of metals. Phys. Rev. B 1988, $37,790$. 
24. Ma, D.; Grabowski, B.; Körmann, F.; Neugebauer, J.; Raabe, D. Ab initio thermodynamics of the CoCrFeMnNi high entropy alloy: Importance of entropy contributions beyond the configurational one. Acta Mater. 2015, 100, 90-97.

25. Oh, H.S.; Ma, D.; Leyson, G.P.; Grabowski, B.; Park, E.S.; Körmann, F.; Raabe, D. Lattice distortions in the FeCoCrNiMn high entropy alloy studied by theory and experiment. Entropy 2016, submitted.

26. Takizawa, S.; Terakura, K.; Mohri, T. Electronic theory for phase stability of nine AB binary alloys, with $\mathrm{A}=\mathrm{Ni}, \mathrm{Pd}$, or Pt and B = Cu, Ag, or Au. Phys. Rev. B 1989, 39, 5792.

27. Laks, D.B.; Ferreira, L.G.; Froyen, S.; Zunger, A. Efficient cluster expansion for substitutional systems. Phys. Rev. B 1992, 46, 12587.

28. Lu, Z.; Wei, S.H.; Zunger, A.; Frota-Pessoa, S.; Ferreira, L.G. First-principles statistical mechanics of structural stability of intermetallic compounds. Phys. Rev. B 1991, 44, 512.

29. Stukowski, A. Visualization and analysis of atomistic simulation data with OVITO-the Open Visualization Tool. Modell. Simul. Mater. Sci. Eng. 2009, 18, 015012.

30. Toda-Caraballo, I.; Wróbel, J.S.; Dudarev, S.L.; Nguyen-Manh, D.; Rivera-Díaz-del-Castillo, P.E.J. Interatomic spacing distribution in multicomponent alloys. Acta Mater. 2015, 97, 156-169.

31. Widom, M. Prediction of structure and phase transformations. In High-Entropy Alloys; Springer: Cham, Switzerland, 2016; pp. 267-298.

32. Song, H.; Tian, F.; Wang, D. Thermodynamic properties of refractory high entropy alloys. J. Alloy. Compd. 2016, 682, 773-777.

33. Dutta, B.; Ghosh, S. Phonon spectra of $\mathrm{Pd}_{x} \mathrm{Fe}_{1-x}$ alloys with transferable force constants. J. Phys. Condens. Matter 2009, 21, 395401.

34. Wang, Y.; Zacherl, C.L.; Shang, S.; Chen, L.Q.; Liu, Z.K. Phonon dispersions in random alloys: A method based on special quasi-random structure force constants. J. Phys. Condens. Matter 2011, 23, 485403.

(C) 2016 by the authors; licensee MDPI, Basel, Switzerland. This article is an open access article distributed under the terms and conditions of the Creative Commons Attribution (CC-BY) license (http://creativecommons.org/licenses/by/4.0/). 